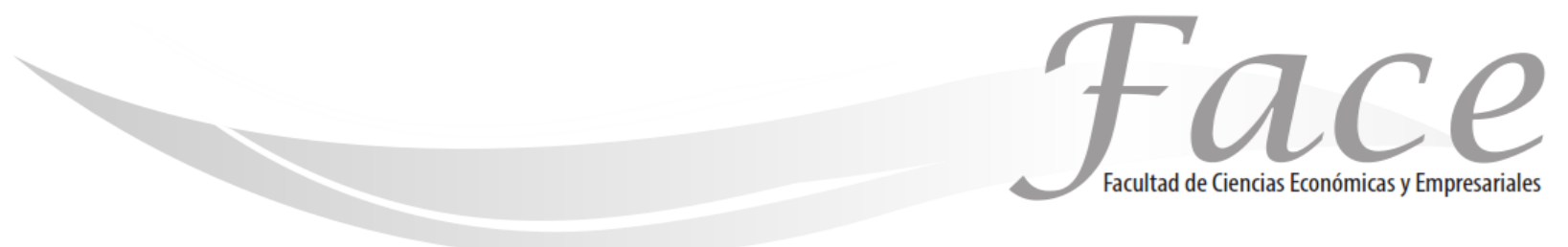

ISSN Impreso: 1794-9920

ISSN Electrónico: 2500-9338

Volumen $18-\mathrm{N}^{\circ} 2$

Año 2018

Págs. 24 - 38

\title{
LA VALORACIÓN DE LOS RECURSOS NATURALES EN LA DISCUSIÓN PARADIGMÀTICA EN CONTABILIDAD
}

\author{
Carlos Alberto Muñoz Restrepo * \\ Enlace ORCID: https://orcid.org/0000-0002-3374-9662 \\ Alba Lucia Gómez Ramírez ** \\ Enlace ORCID: https://orcid.org/0000-0001-6850-7420 \\ Beatriz Elena Moreno Restrepo $* * *$ \\ Enlace ORCID: https://orcid.org/0000-0002-2028-9062 \\ Diego Alejandro Ramírez Tapias **** \\ Enlace ORCID: https:// orcid.org/0000-0002-3609-3320
}

Fecha de Recepción: 30 de Octubre 2018

Fecha de Aprobación: 22 de Diciembre 201

\section{Resumen:}

El presente artículo es derivado del proyecto de investigación que se adelanta actualmente en la Universidad Católica Luis Amigó denominado "Valoración contable ambiental para el Plan de Ordenamiento y Manejo de Cuenca(POMCA) de la quebrada Sinifaná en un trayecto del municipio de Fredonía Fase 1" inicio en Febrero de 2018 noviembre de 2018, y algunos avances derivados de la tesis de maestría en Medio Ambiente y Desarrollo Sostenible de la Universidad Nacional de Colombia denominada "Contabilidad Ambiental en un ecosistema de manglar: Caso delta del Río Atrato", que adelanta una de las autoras. El trabajo se desarrolló a partir de una metodología cualitativa. En él se muestran las consideraciones epistemológicas centrales de los modelos de acumulación del conocimiento, los paradigmas en contabilidad, además, las nuevas perspectivas que se tejen desde la teoría contable y algunas reflexiones en torno al entronque de dichas teorías con la valoración de la naturaleza, los retos que debe asumir el sujeto de conocimiento contable desde el paradigma emergente y las implicaciones directas sobre la vida en la medida en que se asuman posturas distintas frente a la valoración de la naturaleza.

Palabras clave: Valoración Contable Ambiental, Valoración Económica Ambiental, valoración contable, Teoría contable, Métodos De Valoración Contable, Paradigmas Contables

\footnotetext{
* Doctorando en Ciencias Contables, Universidad de los Andes Venezuela- Docente Universidad Católica Luis amigó, Medellín.

Correo: carlos.munozre@amigo.edu.co

${ }^{* *}$ Maestrando en Medio Ambiente y Desarrollo Sostenible de la Universidad Nacional de Colombia Docente Universidad Católica Luis amigó, Medellín. Correo: alba.gomezra@amigo.edu.co

*** Master en Eco Auditoria y Planificación Empresarial del Medio Ambiente, Universidad de Málaga España. Magister en Desarrollo Sostenible Universidad de Manizales Colombia- Docente Politécnico Colombiano Jaime Isaza Cadavid, Medellín - Correo: beatrizelenamoreno@gmail.com

${ }^{* * * *}$ Máster en Auditoría y Gestión Organizacional, Universidad de Puerto Rico (Puerto Rico) - Docente Universidad Católica Luis amigó, MedellínCorreo: diego.ramirez@unisabaneta.edu.co
} 


\section{VALUATION OF NATURAL RESOURCES IN THE PARADIGMÀTIC DISCUSSION IN ACCOUNTING}

Abstract:

This article is derived from the research project currently being carried out at the Luis Amigó Catholic University called Environmental Accounting Valuation for the Plan of Management and Management of the Basin (POMCA) of the Sinifaná creek in a journey from the municipality of Fredonía Phase 1, and Some advances derived from the master's thesis in Environment and Sustainable Development of the National University of Colombia called Environmental Accounting in a mangrove ecosystem: Case delta del Río Atrato, which advances one of the authors. It shows the central epistemological considerations of the models of accumulation of knowledge, accounting paradigms, in addition, the new perspectives that are woven from the accounting theory and some reflections about the connection of these theories with the valuation of nature, the challenges that the subject of accounting knowledge must assume from the emerging paradigm and the direct implications on life insofar as different positions are assumed before the valuation of nature.

Keywords: Environmental Accounting Valuation, Environmental Economic Valuation, accounting valuation, Accounting Theory, Accounting Appraisal Methods, Accounting Paradigms

\section{AVALIAÇÃO DOS RECURSOS NATURAIS NA DISCUSSÃO PARADIGMÁTICA EM CONTABILIDADE}

\section{Resumo:}

Este artigo é derivado do projeto de pesquisa em andamento na Universidade Católica Luis Amigó chamada "contabilidade A avaliação ambiental para 0 Plano de Gestão e Gestão de Bacias Hidrográficas (POMCA) de Sinifaná desfiladeiro em um caminho no município de Fredonia Fase 1" a partir de fevereiro 2018 novembro 2018, e alguns avanços derivados da tese de mestrado sobre Meio Ambiente e Desenvolvimento Sustentável da Universidade Nacional da Colômbia chamado "Contabilidade Ambiental no ecossistema manguezal: delta caso do rio Atrato," que avança um dos autores. $O$ trabalho foi desenvolvido com base em uma metodologia qualitativa. Nele, 0 modelos considerações epistemológicas centro de acumulação de conhecimentos, paradigmas na contabilidade, adição, novas perspectivas são tecidas a partir de teoria da contabilidade e algumas reflexões sobre a intersecção destas teorias para a avaliação de programas de natureza, os desafios que o sujeito do conhecimento contábil deve assumir a partir do paradigma emergente e as implicações diretas na vida na medida em que posições diferentes são assumidas antes da valorização da natureza.

Palavras-chave: Avaliação Contábil Ambiental, Avaliação Econômica Ambiental, Avaliação Contábil, Teoria Contábil, Métodos de Avaliação Contábil, Paradigmas Contábeis 


\section{INTRODUCCIÓN:}

La problemática a la que responde este informe, se enmarca en la discusión acerca de la valoración de los recursos naturales, específicamente en términos de cuál es el método más adecuado de medir y valorar los servicios ambientales que presta un tramo de la quebrada Sinifaná en el municipio de Fredonia Antioquia. Se pretenden abordar unos elementos conceptuales y metodológicos dadas las características esenciales de dicho ecosistema y de los impactos que desde la contabilidad se pueden medir y valorar para el bienestar de la comunidad, como una forma de cumplir con la función social del profesional contable. Obedece a algunas reflexiones conceptuales preliminares en torno a la epistemología de la valoración contable y específicamente la ambiental, que responde al segundo objetivo específico que se deriva de la pregunta ¿cuáles son las metodologías de valoración contable ambiental actuales, y los criterios de reconocimiento contable, más pertinentes para aplicarse al caso?; esto se plantea, por cuanto desde la valoración contable ambiental, la vertiente positiva va en contravía del deber ser de la misma ya que los conceptos propios de la medición y valoración actuales distan de reflejar realidades necesarias que permitan discusiones epistémicas alrededor de la valoración de los ecosistemas hídricos vivos.

La respuesta a esta pregunta ha implicado una serie de exploraciones sobre la discusión acerca de la acumulación del conocimiento, la cual ha pasado por un proceso de desarrollo que recoge aportes derivados de la crisis de los fundamentos de la ciencia, a principios del siglo XX, discusión que dio origen al círculo de Viena y la propuesta del positivismo lógico, hasta los aportes de Hume (1748), respecto de la inducción, asunto revisado posteriormente por Popper (1978), que permitieron, la llegada del concepto de paradigma (luego matriz disciplinar) de Kuhn, (1962), (1970), para consolidar su propuesta de paradigmas y posteriormente, el de programas por parte de Lakatos (1974). Este trabajo recoge los elementos teóricos y la discusión epistemológica sobre la acumulación del conocimiento en la disciplina contable, cuyo eje de discusión, lo constituye el asunto de la valoración

La investigación es de naturaleza cualitativa, pretende la identificación de los fundamentos conceptuales, surgidos de las disputas alrededor de las teorías dominantes en valoración, mediante la identificación de los ejemplares paradigmáticos en la disciplina contable, para deducir el origen de las alternativas de valoración desde la crisis del costo histórico y su incursión en el problema de la valoración de los hechos económicos. La investigación utiliza el método hermenéutico que a través de la interpretación pretende, comprender las circunstancias, para descubrir la consolidación de los métodos de valoración y atribuir un significado en el ámbito de los hechos ambientales.

Inicia la exposición con la identificación del paradigma dominante en contabilidad, a partir del trabajo pionero de Wells en 1976, identificando específicamente los elementos constitutivos de la matriz disciplinar, para argumentar la existencia de una revolución científica en el pensamiento contable. Posteriormente, se revisa detalladamente el proceso revolucionario que gira alrededor de la incapacidad del costo histórico para resolver el problema de la valoración, como consecuencia de los cambios en el entorno, implicando una serie de alternativas que posteriormente emergerán como escuelas de pensamiento, toda esta discusión alrededor de la valoración contable

En el apartado siguiente, se expone, a partir de la noción de teoría rivales acuñado por Lakatos (1970), la emergencia del concepto de ciencia multiparadigmática, concepto aclarado desde el ejercicio de Ritzer, (1975) en el ambito de la sociologia, para ser descrito en la contabilidad por Riahi-Belkaoui, en su publicación de 1981, revisada en 2000. En este periplo, se logra identificar la existencia de un discurso que domina y ha estado presente en la discusión disciplinal, el asunto de la valoración, catalogado por algunos autores como hegemónico Gómez, (2011) 0 como el núcleo (core) por otros (Demski, Fellingham, ljiri, \& Sunder, 2002)

Se cierra la revisión, con la presentación de las posibilidades de valoración del fenómeno ambiental, en el cual se destacan los métodos reconocidos como de "valoración económica", tales como el método de gasto en mitigación, método de reposición, método de la estimación del cambio de productividad y los métodos propuestos por la guía del Ministerio de Ambiente Vivienda y Desarrollo Territorial. (2003), que clasifica en metodologías directas como la valoración contingente y metodologías indirectas (enfoque hedónico, el método de costo de viaje, enfoque de producción de hogares, función de Daño)

Se puede concluir que históricamente se han consolidado cuatro escuelas de pensamiento en valoración y una quinta que constituye un referente ideal Hansen (1966); la Contabilidad a Valor Presente, propuestas por Solomon (July 1961) y Lemke (january, 1966), los cuales posibilitan la valoración de las partidas a efectos de la revelación de los hechos económicos realizados. La valoración de hechos ambientales ha recurrido más a alternativas construidas desde la economía, y se salen de la percepción medición valoración asociadas a la consolidación del proceso contable que tiene como objetos hechos económicos. En este caso se procede a reconocerlos discriminadamente desde los elementos de los estados financieros 


\section{METODOLOGÍA :}

La investigación parte de un diseño metodológico cualitativo, de naturaleza documental, que pretende la identificación de los fundamentos conceptuales, surgidos de las disputas alrededor de las teorías dominantes en valoración, mediante la identificación de los ejemplares paradigmáticos en la disciplina contable, para deducir el origen de las alternativas de valoración desde la crisis del costo histórico y su incursión en el problema de la valoración de los hechos económicos. Dicha identificación, se logra mediando el método hermenéutico, lo cual se hace posible mediante la interpretación, del sentido de las categorías de análisis utilizadas en la revisión. Dado que la naturaleza de los hechos económicos dista de la naturaleza de los hechos ambientales, es necesario estudiar el problema como un proceso social y comprendido desde la visión de sus propios actores, los académicos, desde el análisis de sus teorías. "La investigación cualitativa trata de identificar, básicamente, la naturaleza profunda de las realidades, su estructura dinámica, aquella que da razón plena de su comportamiento y manifestaciones. De aquí que lo cualitativo (que es el todo integrado) no se opone de ninguna forma a lo cuantitativo (que es solamente un aspecto), sino que lo implica e integra, especialmente donde sea importante." (Martínez Miguélez, 2004). De igual forma, es necesario sostener que lo distingue los investigadores en el campo cualitativo es la particular forma de conocer el campo y que es necesario destacar el papel que juega la teoría en la investigación en campos cualitativos. (Ahrens \& Chapman, 2006), para nuestro caso, de la valoración contable.

En su primera fase, el proyecto se embarca en la identificación de los ejemplares más relevantes del campo de la valoración contable, los cuales se identificaron en la etapa de la revolución paradigmática descrita (Wells, Jul., 1976), donde emergen las escuelas de pensamiento clave en valoración contable. Abordar la contabilidad desde este enfoque cualitativo "permite un proceso de indagación flexible, que se mueve entre los eventos y su interpretación, entre respuestas y desarrollo de teoría, con el fin de 'reconstruir' la realidad tal como la observan los actores de un sistema social previamente definido" (Hernández Sampieri, et al, 2010).
La contabilidad en su proceso de representación concibe como fundamento ontológico, la realidad económica del entorno y en la actualidad se enfrenta al reto de hacer visible a través de la representación en reportes contables, otras realidades como la ambiental, para permitir la toma de decisiones y la rendición de cuentas que permitan, no solo la sostenibilidad económica, sino también, la social y ambiental. La investigación utiliza el método hermenéutico que a través de la interpretación pretende, comprender las circunstancias, para descubrir la consolidación de los métodos de valoración y atribuir un significado. La hermenéutica permitirá comprender las representaciones de los hechos ambientales para el desarrollo sostenible como un proceso histórico con su entorno, y proceder a la formulación de metodologías para la presentación de información contable ambiental- no financiera- en pro de la sostenibilidad. Para lograr este enfoque hermenéutico se utilizaron técnicas como la matriz de estrategia de análisis de categorías para diseñar sus instrumentos, lo cual permitió confirmar los supuestos de Ritzer (Aug., 1975)en la concepción multiparadigmatica

\section{RESULTADOS Y DISCUSIÓN :}

\section{Los modelos de acumulación del conocimiento}

Tanto el materialismo mecanicista como el empirismo se constituyeron en los fundamentos anteriores a la nueva etapa de la ciencia, durante finales del siglo XIX y principios del XX. Este periodo es conocido como, "La crisis de los fundamentos de la ciencia y el surgimiento del Círculo de Viena"; la ciencia atraviesa por un periodo de profunda influencia de proposiciones metafísicas, "sin sentido". Para salvar este problema del "sin sentido" aparece una de las mayores conquistas del Círculo, el establecimiento de un criterio de demarcación. Dicho criterio permite separar lo científico de lo no científico, a este primer criterio se constituye en la verificación, si una proposición es verificable, es científica. El "sentido" se reduce a contenido empírico.

En valoración contable ambiental, lo científico obedece a lo ya conocido, al entronque de la contabilidad al sistema financiero, porque es lo tradicional, lo que es posible, medir, valorar e informar, todos los bienes y servicios ambientales que pasan por la esfera del mercado y que son susceptibles de ser informados; para la ciencia no existe la valoración del aire y de las funciones vitales que 
este genera, ni la de la luz del sol, sin el cual no existiría ninguna forma de vida en el planeta; la tierra y el agua solo se miden por hectáreas y se valoran por los bienes que se consumen por el sistema económico o social o por metros cúbicos, no por la calidad y capacidad de los mismos de generar un ambiente sano, un disfrute y un bienestar para nuestra generación y para las venideras. La posibilidad y la capacidad de los ecosistemas de regenerarse una y otra vez y seguir prodigando vida, ahí está el paradigma emergente, el que debe ser asumido por el contable actual, la naturaleza debe medirse y valorarse por esa posibilidad futura de prodigar vida, no por la naturaleza muerta que es propia del paradigma científico actual y que sólo mide y cuenta toneladas de madera, metros cúbicos de agua distribuida, peces que llegan a la economía y luego a la mesa del consumidor final entre otros.

Este tipo de reflexiones de naturaleza filosófica, también permiten descubrir la insuficiencia del lenguaje común para elaborar enunciados descriptivos 0 proposiciones susceptibles de cientificidad, nos referimos al primer encuentro con el problema de la verdad de los enunciados científicos. La denominada paradoja de la predicación científica, o la paradoja del cretense mentiroso; dicha conquista nos permite reconocer la necesidad de distinguir entre lenguaje objeto y metalenguaje, es decir un lenguaje que haga posible la significación de los enunciados sin caer en la paradoja. (Tarski, 1999)

Estos aportes del círculo, con una profunda influencia del empirismo de David hume,

La conceptualización del empirismo y del positivismo acerca de la naturaleza del proceso de acumulación de conocimiento se ha sustentado siempre en el proceso de inducción. Este principio señala, tal como lo plantea Hume, que si observa una cierta regularidad en los procesos naturales (incluida la naturaleza humana), entonces es posible generalizar a partir del establecimiento de una ley (Mercado-Domenech, 2008, pág. 5)

Se asume el trabajo de Círculo en la necesidad de formular las proposiciones en términos de observabilidad de los enunciados que permiten construir las proposiciones, la cuál ha de recurrir a un procedimiento de verificabilidad, que reduce las proposiciones científicas a proposiciones sobre sensaciones. A este proceder tradicionalmente se le ha tachado de "positivismo rayano".

En este mismo escenario, la aparición de Sir Karl Raymond Popper, pone en evidencia las limitaciones de las inferencias probabilísticas que fundamentan las leyes científicas, la construcción de una ley científica, que se supone absolutamente verdadera, en principio, se levanta sobre la base de un procedimiento de generalización de las observaciones, en una proposición que funge como ley descriptiva general, válida para todo tiempo y lugar. gran mérito de Popper fue encontrar, en su metodología de la falsación, un procedimiento que nos permite, reconocer con absoluta certeza, que es más viable falsar que verificar.

Popper (1974) señala que es precisamente esa búsqueda de una base firme e incontrovertible la fuente de los problemas. Hace un análisis sobre la reflexión de Hume (1927-1960) acerca de la inducción y coincide con él en que no es posible que partiendo de la observación de una serie de casos reiterados de una relación determinada se llegue a una conclusión válida acerca de casos aún no observados; es decir, no se justifica desde el punto de vista lógico la inferencia (Mercado-Domenech, 2008, pág. 9)

La clásica proposición que ha servido de ejemplo, "todos los cisnes son blancos", le permite reconocer que mediante un conjunto de observaciones particulares que corroboran la proposición, hace posible la generalización de esta en una ley científica. Generalmente este procedimiento, inductivo, a partir de la observación que posibilita la muestra sobre una base poblacional, logra ser controvertida al reconocer que una sola observación que niegue la proposición, falsa la ley al invalidar el procedimiento inductivo. En este sentido, el hecho de que en la naturaleza sean mayores las relaciones y tejidos ocultos que lo que se percibe a través de los sentidos (propios de la ciencia) inhibe una valoración real de los bienes y servicios que se proveen para bienestar de todas las especies y de la supervivencia de los diferentes ecosistemas, ya que está desconociendo las razones que posibilitaron el mismo, el tiempo que tardo en generarse, el tiempo que se necesitó para favorecer la capacidad de ofertar múltiples servicios ambientales y todos al mismo tiempo.

Mientras el circulo, se constituye en el discurso dominante durante esta etapa de la ciencia, y permea la concepción de ciencia en la casi totalidad de los criterios de cientificidad, aparece en los años 60 's, un físico, aporta importantísimas innovaciones al proceder científico y concepto de ciencia, mediante su trabajo histórico y filosófico. Thomas S Kuhn en 1962 publica "La estructura de las revoluciones científicas", el primer intento en construir un modelo de acumulación y crecimiento del conocimiento es producto de su esfuerzo.

Durante su obra, se perciben cambios en la noción de paradigma, pudiéndose identificar como "el conjunto de supuestos compartidos por una comunidad" (Osorio, 2014, pág. 4). Asi mismo, podemos descubrir que reconoce el cambio de denominación de paradigma por el de matriz disciplinar en el poscriptum de 1970 (Wells, Jul., 1976). No obstante, la posibilidad de identificar la matriz, depende de la identificación de la comunidad de académicos (Wells, 
Jul., 1976) citando a (Kuhn, 1962)1, sin recurrir, lógicamente, a la membresía previa a un paradigma. ¿Ahora bien, cual es el contenido de una matriz disciplinaria?, Wells, la describe la siguiente forma, interpretando a Kuhn:

1. generalizaciones simbólicas - fácilmente entendidas y representaciones simbólicas indiscutibles comunes a la disciplina [p. 182];

2. compromisos compartidos - creencias que ayudan a determinar lo que se aceptará como explicaciones o soluciones [p. 184];

3. los valores-las diversas cualidades que los miembros de la comunidad esperan en el trabajo de sus colegas [pp. 184 - 186]; y

4. ejemplares -el problema concreto- soluciones a las que se enfrentan los estudiantes que entran en la comunidad y cuáles son, por ejemplo, cómo deben buscar soluciones [pág. 187] (Wells, Jul., 1976, pág. 473) citando a (Kuhn, 1970)

Todas estas disquisiciones epistemológicas se aplican perfectamente en la contabilidad ambiental, infortunadamente desde el paradigma dominante no se aplican estas, si desde el paradigma emergente con el ánimo de contribuir a medir y valorar la naturaleza no desde la ficción que muestra la óptica rentística de los bienes y servicios ambientales sino desde el verdadero valor que deben tener estos para la vida. Desafortunadamente son pocos los sujetos de conocimiento contable que se atreven aún a romper las fronteras del conocimiento hegemónico para entrar a proponer visiones holísticas, complejas, interrelaciones e interdependencias entre el sistema natural, el sistema económico y el sistema social, que, si se analizan a fondo, tienen mayor valor que el que ofrece la economía y en síntesis el mercado en estos momentos.

\section{Los paradigmas en contabilidad}

La construcción paradigmática en contabilidad, es decir, desde la metodología propuesta por Thomas Kuhn, se inaugura en el campo contable con el trabajo pionero de Wells (1976), seguido por las construcciones de RiahiBelakoui, (1981) y su actualización en Riahi-Belakoui, (2000).

En nuestra disciplina constituye un esfuerzo de MC Wells en el año 1976, trabajo a partir del cual, La American Accounting Asociation, publica el Statement on Accounting Theory and Theory Acceptance", en 1977. Wells identifica

\footnotetext{
${ }^{1}$ En su momento, Wells, identifica claramente dicha comunidad en términos de los académicos de la American Accounting Association, the Association of University Teachers of Accounting of the United Kingdom, the Accounting Association of Australia and New Zealand, the Research Division of the AICPA and the Australian Accountancy Research Foundation (pág. 473)
}

que la técnica básica de la teneduría de libros, domino por 500 años y que solo recientemente, el siglo XX, la doctrina del costo histórico logra dominar en la academia y la práctica (Jul., 1976, pág. 473). El trabajo pionero de Wells, nos permite identificar, específicamente los componentes del paradigma dominante, en su época, en la contabilidad

Figura 1 Matriz disciplinar identificable a partir de los años 40 's en la comunidad académica anglosajona

\begin{tabular}{|c|c|}
\hline $\begin{array}{ll}\text { COMPONENTES } & \text { DE } \\
\text { MATRIZ } & \text { DISCIPLINAR } \\
\text { KUHN } 1970 & \end{array}$ & $\begin{array}{l}\text { MATRIZ DISCIPLINAR } \\
\text { CONTABILIDAD, } 1976 \text { (década de los } \\
40 \text { ) }\end{array}$ \\
\hline $\begin{array}{l}\text { generalizaciones } \\
\text { simbólicas }\end{array}$ & $\begin{array}{l}\text { - La ecuación de doble entrada, } \\
\text { - } \text { Representaciones de } \\
\text { ingresos, } \\
\text { Clasificación de active } \\
\text { corriente / activo fijo } \\
\text { - Cálculos de capital de trabajo, } \\
\text { - tasa de rendimiento y Ratios } \\
\text { de deuda y patrimonio }\end{array}$ \\
\hline compromisos compartidos & $\begin{array}{l}\text { - Los principios de realización y } \\
\text { asociación de ingresos con } \\
\text { costos y gastos, } \\
\text { - la noción de continuidad y } \\
\text { - la base de costos para la } \\
\text { valoración de activos }\end{array}$ \\
\hline los valores & $\begin{array}{l}\text { Conservadurismo, consistencia } \quad \text { y } \\
\text { materialidad }\end{array}$ \\
\hline ejemplares & Libros de texto y demás publicaciones \\
\hline
\end{tabular}

Fuente: Elaboración propia a partir de (Wells, Jul., 1976).

En este periodo, los investigadores acuerdan en seguir un conjunto de principios contables desde 19332. Sin embargo, la formalización de las reglas no elimina las contradicciones y los conflictos. Los investigadores ven ciertas anomalías en la aplicación del costo histórico, como en la valuación de inventarios a la regla de costo o valor de mercado el más bajo, y la aplicación del cargo de depreciación del costo de los activos. Esto genera variadas críticas, pues las alternativas son aplicables en contextos específicos y pueden ser explicadas por la particularidad de hechos en cada caso. Además de ello, aparece una claridad importante en la precisión de la naturaleza del proceso contable; la valoración ha de precisarse en el tiempo, esto es, se trata del momento del reconocimiento y el momento de la revelación, y estamos refiriendo la valoración de hechos económicos realizados.

\footnotetext{
${ }^{2}$ En el año 1933, los principios logran fuerza normativa con la publicación por parte de la New York Security Exchange - NYSE. Adicionalmente el asunto venia tratándose, como puede verificarse en la correspondencia publicada por La American Institute of Accountants y NYSE.
} 
Descripción del proceso revolucionario en contabilidad El proceso revolucionario, específicamente el periodo de crisis es caracterizado por Kuhn por siguientes etapas (Wells, Jul., 1976, pág. 471)

1. Reconocimiento de anomalías

2. Un período de inseguridad

3. Desarrollo de conjuntos alternativos de ideas

4. Identificación de escuelas de pensamiento

5. Dominio de las nuevas prácticas o ideas

\section{Reconocimientos de anomalías y periodo de inseguridad}

El descubrimiento comienza con la conciencia de la anomalía, es decir, con el reconocimiento de que la naturaleza ha violado de alguna manera las expectativas inducida al paradigma que rigen la ciencia normal [o la práctica convencional] (Kuhn, 1970, pp. 52-53). Según Wells, Las críticas a la matriz disciplinaria que cristalizò el costo histórico (Paton [1922], Sweeney [1936] and MacNeal [1939]) tuvieron consecuencias, entre los 60's y 70 's entre académicos, hombres de negocios y las cortes ${ }^{3}$.

Se refiere entonces la sentencia de Kuhn, cuando se enfrentan a la anomalía [los científicos] inventarán numerosas articulaciones y modificaciones ad hoc de su teoría (Muñoz-Restrepo, 2018) con el fin de eliminar cualquier conflicto aparente [Kuhn, 1970, p. 78]. (Wells, Jul., 1976, pág. 475)

Aquí es importante destacar que, en el periodo de inseguridad que corresponde a la etapa por la cual atraviesa el proceso revolucionario, se introducen modificaciones Ad Hoc, para eliminar el conflicto o anomalía; Así, el cuerpo profesional demanda aferrarse estrictamente a los pronunciamientos. Las soluciones ad hoc que surgen durante un período de crisis tienen una consecuencia de largo alcance; hacen posible contemplar reglas que previamente habría sido inaceptable. Es decir, "(...) por la proliferación de versiones de la [matriz disciplinaria] crisis, afloja las reglas de resolución de enigmas normales en formas que, en definitiva permita a la nueva [matriz disciplinaria] surgir " (Wells, Jul., 1976) citando a (Kuhn, 1970), p.e. existen anomalías intratables, como por ejemplo el sistema basado en el costo histórico falla al considerar el cambio en el precio de los activos y en el poder de compra de la unidad monetaria, especialmente en periodos de inflación. Frente al particular

Es, creo, sobre todo en períodos de crisis reconocidos que los científicos han recurrido a análisis filosófico como un dispositivo para desbloquear los misterios de su campo (...), el trabajo puede llevarse a cabo utilizando el

${ }^{3}$ Para la discusion, puede verse Briloff's Un accountable Accounting [ 1972] and Chambers Securities and Obscurities [1973]. paradigma como modelo, las reglas y los supuestos no tienen que ser explícitos. Pero eso no quiere decir que la búsqueda de supuestos (...) no puede ser un medio eficaz para debilitar el agarre de la mente a tradición y proponer una nueva base (Wells, Jul., 1976) citando a (Kuhn, 1970).

Curiosamente es en lo periodos de crisis donde aparece la preocupación por los fundamentos de la contabilidad, aparece la necesidad de discutir filosóficamente4. Especialmente, los problemas y relaciones de la naturaleza y de las formas como esos recursos se generan y llegan al mercado con el fin de brindar una información más concreta y real de los bienes y servicios ambientales. Una vez más, ha habido mucho interés por los fundamentos teóricos de la Prácticas de Contabilidad5, que es necesario enlazar con las problemáticas ambientales. Además, es posible distinguir dos desarrollos como los mencionados, el primero es la búsqueda de supuestos. De particular interés aquí son

- Littleton's The Structure of Accounting Theory [1953],

- Moonitz's The Basic Postulates of Accounting [1961],

- the American Accounting Association's A Statenmento f Basic Accounting Theory [1966],

- ljiri's The Foundations of Accounting Measurenment [1967] 6

El otro desarrollo que es particularmente notable a lo largo de este período es la preocupación por los principios y la construcción de la teoría general, comenzando quizás con

- Chambers' "Blueprint for a Theory of Accounting" [1955],

- notable contributions or comments by Mattessich [1957],

- Devine [1960],

- Chambers [1963],

- Vatter [1963] and

- Sterling [1970] followed

\footnotetext{
${ }^{4}$ el AICPA en 1971, reconoció la necesidad de una declaración de, y puso en marcha un estudio de, los "objetivos "de los estados financieros, esto tendrá una profunda influencia en el "core" de la disciplina, es decir en el asunto dela valoración, ¿valorar con qué objetivo?

5 como en el caso de Chatfield, 1974, Chapter 16; Hendriksen,1970, Chapter2, citados por (Wells, Jul., 1976)

${ }^{6}$ Para más ejemplos, incluyendo los cambios desde y hacia la depreciación acelerada y hacia y desde los valores de inventario LIFO, se debe consultar a Chambers [1973, p p. 93-103].
} 
Durante los 60 y 70's El Accounting Principles Board - APB y otros comités, proponen modificación de las normas para hacer frente a las anomalías y críticas.

\section{Propuestas alternativas y su evaluación}

Una de las consecuencias de la discusión filosófica ha sido la emergencia y refinamiento de alternativas a la matriz disciplinaria, por ejemplo, a la valuación de activos al costo histórico así, podemos identificar en los siguientes ejemplares, intentos para superar los defectos del costo histórico (Wells, Jul., 1976)

- Edwards and Bell [1961] and

- Chambers [1966]; others include

- Sprouse and Moonitz [1962],

- Mattessich [1964] and

- Mathews [1965]

Ambos son una reacción natural al reconocimiento de anomalías y un paso vital en la selección de una nueva matriz disciplinaria. Aquí hay algunas otras características de la etapa evaluativa que se describieron en Kuhn y que puede verse también en la contabilidad. Kuhn llamó la atención sobre las similitudes de la etapa de evaluación para el período pre-paradigma. Es la etapa en la que "los debates frecuentes y profundos sobre los métodos legítimos, los problemas y las normas de la solución" se llevan a cabo, a pesar de que "éstos tienden a definir las escuelas en lugar de producir un acuerdo" (Wells, Jul., 1976) citando a (Kuhn, 1970), Así entonces la emergencia de escuelas es una muestra de la falta de consenso

En la contabilidad, esta etapa ha estado marcada por los debates acerca de la admisibilidad de los datos relativos a los acontecimientos externos a la empresa y los datos sobre la base de las intenciones de los directivos ${ }^{7}$. Frente a esta etapa de desarrollo del paradigma, es claro al afirmar que las distintas propuestas parezcan igual de relevantes, pero que la actividad científica posteriormente, hace reconocida (Wells, Jul., 1976) citando a (Kuhn, 1970) Esto tal vez, es la razón por la que muchas de las propuestas han surgido en los últimos años. Estos incluyen sugerencias para

\footnotetext{
${ }^{7}$ La presentación de los estados de flujo de efectivo, las ganancias por los cálculos de acciones, el reconocimiento de dos tipos de gananciales, los "gains holding" y los gananciales operativos, la admisibilidad de métodos de valoración contable como valor neto de realización (valores de salida), o costos de reemplazo (valores de entrada). Edwards y Bell, etc. Asi como, El aumento de los problemas, como El reconocimiento de las tenencias de moneda extranjera, La presentación de informes para las empresas diversificadas, Contratos a largo plazo y Proyectos de desarrollo de la tierra; La legitimidad de las asignaciones de costos; y Reconsideración de las normas que las soluciones deben cumplir, como la objetividad, la independencia y la libertad de prejuicios.
}

- La publicación de los estados financieros de varias columnas y pronósticos (Glover, 2008);

- El desarrollo de la contabilidad del comportamiento; $y$, en un nivel diferente,

- La investigación de largo alcance en los movimientos de precios de acciones y su teoría de la información y

- El análisis de costo-beneficio para el suministro de información financiera

- La discusión sobre los fundamentos de la disciplina (Demski, Fellingham, ljiri, \& Sunder, 2002)

En la naturaleza, el costo histórico muestra otro sesgo y es que no se tiene en cuenta el bien que entrega la naturaleza, generando un desbalance biológico es decir se rompe el balance de masa al recolectar la cosecha y no devolver a esta por el bien aportado, generándose repetidamente un desequilibrio en el sistema natural con evidentes consecuencias en el sistema económico y en el sistema social. Desde el paradigma emergente, se haría necesario empezar a medir y valorar el pasivo ambiental generado por años de apropiación de dichos bienes, sin retribución positiva aparente a la misma.

\section{Escuelas de pensamiento}

Pueden identificarse cuatro escuelas de pensamiento en la medición/valoración de activos, No es descabellado pensar que, no obstante la perspectiva multiparadigmatica, y el asunto de la inconmensurabilidad, el asunto central de la contabilidad o por lo menos, hegemónico (Gomez, 2011), es precisamente este, el problema de la valoración. (Wells, Jul., 1976, pág. 478). la perspectiva paradigmática, sobre el asunto valorativo, se describe en términos de las cuatro escuelas de pensamiento y sus ejemplares en el apartado que aborda la perspectiva multiparadigmatica, dichas escuelas pueden enumerarse como:

1. Contabilidad Ajustada al nivel de precios

2. Contabilidad a costo de reemplazo

3. Contabilidad a Valor de Privacion

4. Contabilidad a valor neto realizable.

Sin embargo, "No se ha enumerado la quinta propuesta. A pesar de que ha sido discutido por Hansen [1966], que no parece haber ganado el sustento como una alternativa operacional. se ha discutido más como un ideal contra el cual las alternativas se pueden evaluar. Por ejemplo, Solomons [1961] y Lemke [1966]". (Wells, Jul., 1976, página 478). Es decir, Contabilidad a Valor Presente.

Los debates en la transición de una matriz disciplinar, sobre la base de la investigación a priori, han tenido otras características. 
La adopción de una nueva matriz disciplinaria, normalmente, requerirá un cambio fundamental en el punto de vista que los teóricos tienen del mundo. Por lo tanto, en la contabilidad ambiental se han producido cambios que implican

- La opinión de que el valor de la unidad monetaria es estable ha cambiado a la aceptación de la opinión de que es variable;

- La opinión de que el momento de realización debe ser el momento de reconocimiento de las ganancias, está dando paso a la idea de que otra evidencia de ganancias es admisible; y

- La idea de que sólo las transacciones reales dan lugar a datos objetivos está dando paso a una noción menos restringido de objetividad. ${ }^{8}$

Teniendo en cuenta a la naturaleza, estas 4 escuelas de pensamiento, son totalmente aplicables, el rol del sujeto de conocimiento contable es ahondar en las diversas complejidades de la naturaleza para aportar un valor diferente al que se muestra hoy en la contabilidad financiera ambiental. Estos mecanismos de medición y valoración dependen de las necesidades de información del sujeto que quiere medir y valorar, ya que para un inversor la naturaleza tiene un valor distinto al de un disfrutador del paisaje, por lo tanto las subjetividades deben convertirse en intersubjetividades que permitan generan diálogos francos y abiertos frente al deber ser de los mismos mecanismos de valoración porque lo que si es cierto es que tanto inversor como disfrutador del paisaje, tienen un mismo objetivo en últimas, seguir viviendo, para ello se requerirá, dentro del paradigma emergente, nuevas conciencias aportantes a la vida y al bienestar de todos.

La ciencia en perspectiva multiparadigmatica ritzer, 1975, justificación frente a confrontación

De acuerdo con Lakatos (1970), un programa de investigación se juzga a partir de su comportamiento comparado con programas rivales. La conciencia de nuevas variables extrañas generalmente se da en torno a la competencia entre teorías rivales; el investigador no se percata de qué variables debe controlar hasta que otra explicación sugiere los aspectos que debe considerar con más cuidado para decidir cuál explicación es la que mejor da cuenta de los hechos. Lakatos (1968b) citado por (Mercado-Domenech, 2008) asevera que no es tan importante el choque entre teoría y datos como la

\footnotetext{
${ }^{8}$ Considerado en su momento, pues hoy día, el problema ha virado hacia la preocupación sobre el realismo externo como presupuesto ontológico de los números contables, como puede verificarse en la interesante discusión (Lee, 2009), (Mattessich, 2003), (Macinstosh, Sheare, Thorton, \& Welker, 2000), y otros.
}

competencia entre las teorías rivales. La actitud rigurosa no implica la supresión instantánea de una teoría, sino la exploración seria y crítica de sus posibilidades frente a otras opciones de explicación. Tal como señala Weimer, "en la mayoría de los casos en la práctica científica actual, el medio más efectivo de crítica disponible para un investigador es permanecer comprometido con una posición para poder articularla plenamente y explorar sus consecuencias"(Weimer, 1979, p. 49). (MercadoDomenech, 2008, pág. 10)

\section{Visión de Ritzer de los múltiples paradigmas aplicados a la contabilidad}

El auge y caída de los paradigmas es el resultado de factores políticos desde la perspectiva que un paradigma gana a otro por muchas razones, entre las que tenemos, (Kuhn, 1970), exactitud, el alcance, la sencillez, la fecundidad y gusto.

George Ritzer en favor de la primera perspectiva sostiene que la emergencia de un paradigma es esencialmente un fenómeno político (Ritzer, Aug., 1975, pág. 156), asegura que los paradigmas estan determinados por hechos que los hace políticamente dependientes

la emergencia de un nuevo paradigma, o la falla de otro en emerger, puede ser atribuido a factores políticos en lugar de los relativos meritos "científicos" de los paradigmas. Esto no implica que los meritos científicos de un paradigma son importantes para su éxito. (Ritzer, Aug., 1975, pág. 157)

en este orden, se puede sostener que, el paradigma sostiene el control de los más importantes journals en un campo, y sus líderes en un campo quienes sostienen el paradigma $^{9}$, buscando legitimarlo. Siendo los paradigmas políticamente dependientes, Ritzer define el paradigma como

Un paradigma es la imagen fundamental de la materia dentro de una ciencia. Sirven para definir, que puede ser preguntado y que reglas pueden ser seguidas en la interpretación de las respuestas. Es la unidad más amplia de consenso y sirve para distinguir una comunidad científica de otra. Subsume, define, e interrelaciona los ejemplares, teorías, métodos e instrumentos que existen en él.

\footnotetext{
9 Pudo observarse, la participación de los journals en la consolidación de los ejemplares a través de su publicación, por ejemplo, The Accounting Review, participa con 7 de las 12 publicaciones, que no son libros. Por su parte AICPA y AAA participan con 14 de las 20 publicaciones en formato de libro.
} 
Así, los componentes básicos de un paradigma emergente son:

- Un ejemplar, o pieza de trabajo que soporta un modelo, para aquellos que trabajan con el paradigma

- Una imagen de la materia "objeto"

- Teorías

- Métodos e instrumentos

Es entonces que analizamos la comunidad científica con las asunciones que, no hay un paradigma dominante, y cada (sub) paradigma (subcomunidad) se esfuerza por aceptar o dominar dentro de la disciplina. Alrededor del asunto hegemónico de la valoración e contabilidad, han surgido otras materias iguales o más relevantes, que tiene cabida en una perspectiva multiparadigmática. Analicemos esto

Contabilidad: una ciencia multiparadigmatica RiahiBelkaoui, 2000

Ahora bien, entrando en materia de contabilidad, encontramos antecedentes de esta construcción, en primer lugar en (Wells, Jul., 1976) y posteriormente en la publicación de la American Accounting Asociation, (AAA) en 1977 bajo su Statement of Accounting Theory and Theory Acceptance (Declaración de teoría contable y aceptación de la teoría). Por su parte (Riahi-Belkaoui, 2000), realiza la siguiente construcción en términos de la visión multiparadigmática en contabilidad. la construcción del dichos paradigmas, es revisada en el texto Accounting Theory por Riahi-Belkaoui, en el año 2000, que en su capítulo 10 logra establecer, a partir de la proposición multiparadigmática de Ritzer (Aug., 1975).

En el campo específico de la Valoración en contabilidad, la descripción de la discusión sobre la valoración contable en Riahi-Belkauoi, es recogida por lo que denomina "paradigma de ingreso verdadero", estos son sus ejemplares y sus teorías;

\section{Ingreso verdadero deductivo}

1. Paton, W.A 1922; Accounting Theory. New York: The Ronald Press

2. Canning, JB 1929; The Economics of Accountancy. New York The Ronald Press

3. Sweeney, Henry W 1936; Stabilized Accounting. NY Haper \& Row.

4. Macneal, Kenneth 1939; Truth in Accounting Philadelphia. University Pensilvania Press
5. Alexander, Sidney 1950; Income Measurement in a Dynamic Economy. Five Monographs of Bussiness Income. (NY :The Study Group on Business Income. AlCPA)

6. Edwards, EO y Bell, PW 1961; The Theory and Measurement of Business Income. Berkeley Unirversity of California Press.

7. Moonitz, Maurice 1961; The Basic Postulates of Accounting. Accounting Research Study, No1 New York AICPA

8. Alexander Sidney S, 1961; Income Measurement in a Dynamic Economy. (rev. David Solomons, In Studies in Accounting Theory, (eds) W.T Baxter and Sidney Davinson (Homewood, I. LI.:Richard D.Irwin, 1962)

9. Sprouse, RT and Moonitz, Maurices 1962 Acoounting Research study No 3 A tentative Set of Broad Accounting Principles For Business Enterprise , New York AICPA

\section{Teorías o escuelas de pensamiento}

1. Contabilidad Ajustada al nivel de precios PriceLevel-Adjusted (or current-purchasing-power) Accounting

2.

a. Jones[ 1956] Jones Ralph Coughenour, The Effects of price - level Changes (saratosaFl.: AAA)

b. Mason [1971] Mason, Perry. Price level changes of Financial statements (Sarasota, Fl.: AAA)

3. Contabilidad a costo de reemplazo ReplacementCost Accounting

a. Edwards\& Bell [1961] Edwards, EO y Bell, PW 1961; The Theory and Measurement of Business Income. Berkeley Unirversity of California Press

b. Mathews [1965] Mathew Russell, L, "price-level Accounting useless information", Journal of Accounting Research (Spring 1965) pp.133-155

c. Gynther[ 1966] Gynther, RS, "Accounting for Price level changes: Theory and Procedures (NY: Pergamon, 1966)

d. Revsine [1973] Revsine, L. Replacement - cost Accounting. Englewood cliffs, NJ: Prentice Hall, 
4. Contabilidad a Valor de Privacion Deprival-Value Accounting

a. Baxter [1967] Baxter, WT, "Accounting Values: Sale price vs Replacement Cost", Journal of Accounting Research (Autum, 1967), pp208-14

b. Wright[ 1970] Wright, F. K, "A theory of financial accounting" journal of business finances, (Autum, 1970) pp. 51-69

c. Stamp [1971] Stamp,Edward, "income a value determination changing price levels: An Essay toward a theory" The accountants magazine (june, 1971) pp 277-92

d. Whittington[1 974] Whittington, Geoffrey, "Asset Valuation, income measurement and accounting income", Accounting Business and research, (Spring 1974) pp 96-101

5. Contabilidad a valor neto realizable. Continuously Contemporary (Net-realizable-value) Accounting

a. Chambers [1966] Chambers, R J, Accounting, Evaluation, And Economy Behavior (Englewood cliffs, $\mathrm{NJ}$ : Prentice Hall, 1966)

b. Sterling [1970] Sterling, Robert R, "On Theory Construction and Verification", The Accounting Review (January, 1971) pp12-29

6. Contabilidad a Valor Presente. Present -Value Accounting

a. Solomon, David, "Economic and Accounting Concepts of Income", The accounting Review , (July 1961) pp37483

b. Lemke, Kenneth W., "asset valuation and Income theory", The Accounting Review, (january, 1966) pp33-41
La valoración de los recursos naturales: paradigma dominante vs el paradigma emergente.

Desde el paradigma dominante los recursos naturales son valorados como un bien que prodiga la naturaleza para el disfrute de los seres humanos, al ser conceptualizado como recurso se puede reemplazar fácilmente uno por otro (recurso natural vs recurso manufacturado), esto es, cambiar un bosque por un edificio, 10 litros de agua, por medio litro de gasolina. Supuestamente se está cumpliendo con el equilibrio al aprovechar un bien y reemplazarlo por otro, pero en la realidad ocurren cosas distintas, si se consume el litro de agua, se está satisfaciendo una necesidad humana de mitigar la sed, si se cambia por gasolina, no se cumple la función vital. Frederick Soddy en 1921, formulaba que en la naturaleza siempre hay un déficit energético por cuanto la energía se utiliza para el uso vital y para el uso laboral y está en déficit en el sentido en que no puede volverse a aprovechar, entonces la pregunta que cabe hacerse desde el paradigma dominante es porque el hombre construyo un concepto de utilidad para apropiarse de unos bienes que no generó él, sino la naturaleza (el sol y las plantas a través de la fotosíntesis) y el mismo hombre. Esto denota una visión sesgada con respecto a las funciones vitales que cumple la naturaleza. Desafortunadamente la contabilidad y ante todo la contabilidad ambiental, está sumergida aún en este paradigma, negándole a la naturaleza la posibilidad de seguir prodigando bienestar a todos por siempre.

Desde el modelo de contabilidad establecido por Colgaap, se establece la valoración solo en unidades monetarias, (el paradigma hegemónico descrito) solo a bienes que pasan por la esfera del mercado, los bienes que no se han establecido como "escasos" no pueden ni deben ser contabilizados como activos, y por si fuera poco al ser difícil de establecer los pasivos ambientales (deudas adquiridas por su aprovechamiento, sin cumplir el principio de equilibrio y reciprocidad establecido por Franco (2018) con respecto a los recursos naturales, por lo tanto establecer el patrimonio natural de los bienes y servicios ambientales que prodiga la naturaleza es casi que imposible. (Gómez A. 2018) Desde el modelo Sistema de Contabilidad Ambiental Económica SCAE (2016) de las Naciones Unidas (UN), se muestra como estos pueden ser medidos y valorados en unidades físicas, en unidades monetarias, través de indicadores. Como se muestra en el cuadro 1. 
CUADRO 1. SISTEMA DE CUENTAS SATÉLITES AMBIENTALES

\section{SCAE $\begin{aligned} & \text { SISTEMA DE CONTABILIDAD } \\ & \text { AMBIENTAL Y ECONÓMICA }\end{aligned}$}
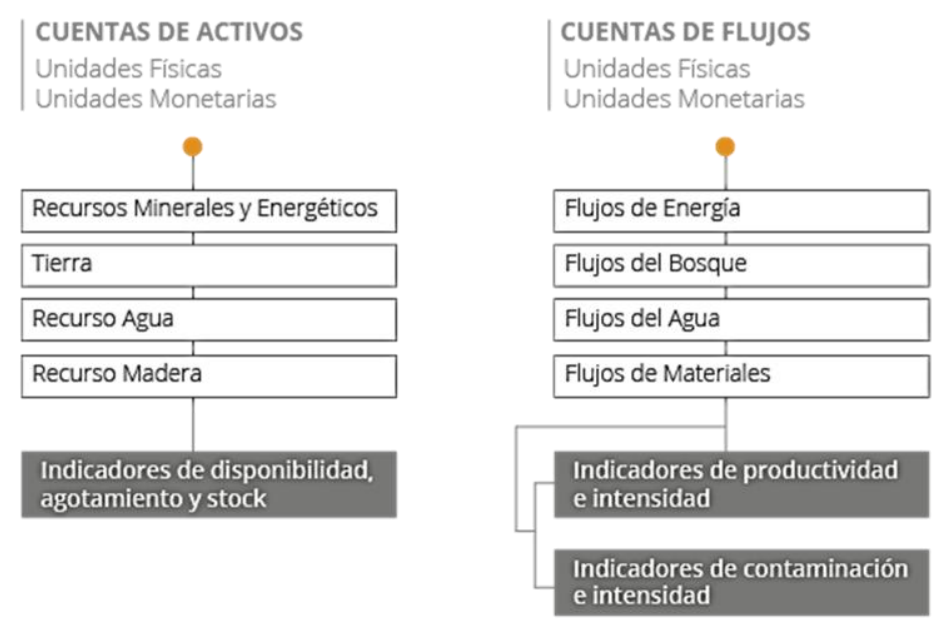

Fuente: Dane (2017) Cuenta satélite ambiental

Dentro del paradigma dominante, esta información y método de medición y valoración sólo muestra los bienes que ingresan al sistema económico, con respecto a las cuentas físicas del suelo, el sesgo se genera porque es lo mismo una hectárea de tierra sembrada en pasto que una hectárea de bosque, desconociendo los verdaderos y múltiples bienes y servicios ambientales que aporta este. Desde el paradigma emergente, los bienes y servicios ambientales no se transan en el mercado, los mismos se deben valorar desde el bienestar que se genera. La contabilidad desde esta perspectiva está en mora de información que sea útil para la conservación del planeta en las condiciones actuales, es necesario contabilizar los bosques, aguas, flora, fauna y suelo, aire, desde la posibilidad de generar vida, lo que hace el paradigma dominante, parafraseando a (Machado 2018) (ent.per), es contabilizar la naturaleza muerta no la naturaleza viva. Es pertinente reconceptualizar la riqueza, la propiedad, la rentabilidad y otorgarle valores de uso a la vida, antes que a la muerte (madera, peces, contaminación etc).

\section{El Fenómeno Ambiental, los Métodos de Valoración Contable}

Existe una disputa respecto de la valoración de los fenómenos ambientales. Dicha disputa se da entre las disciplinas que se abrogan el derecho de valoración. Algunos teóricos del asunto, lo resuelven bajo la rúbrica de métodos de valoración económica, o bien valoración económica ambiental. La contabilidad ambiental forma parte inherente de Contabilidad aplicada en busca de las relaciones entre las organizaciones y el medio ambiente, el medio ambiente de una empresa puede denominarse como su entorno natural, vital, cultural y social. (Cuesta, 2004).

La contabilidad ambiental se basa en una contabilidad la cual tiene como objetivo la identificación y medida de la contribución social la cual permite a la empresa cuantificar el resultado social. Para llegar a esta evaluación es necesario realizar una medida monetaria de los costos ambientales, donde se estimen dichos costos, si bien se incurren a diario en ellos, estos están inmersos en los costos tradicionales, lo que hace necesario la revelación y el reconocimiento en los informes financieros por separado. 
Existen limitaciones que influyen en el proceso de valoración del medio ambiente, como lo son la falta de aceptación para monetizar aspectos de la vida humana y su entorno, la inexistencia de tasas sociales de descuentos, las cuales ayudarían a estimar los valores de los daños medioambientales, la falta de conocimiento de los daños generados en el ecosistema y que generan un gran impacto ambiental. Por lo anterior se genera la dificultad en la valoración monetaria de los costos y beneficios ambientales en cuanto al uso de los recursos naturales.

\section{Valoración económica ambiental}

método de gasto en mitigación. Este método intenta cuantificar lo que las personas están dispuestas a gastar para evitar la el impacto al medio ambiente. El cual muestra que los individuos realizan los gastos en mitigación si el beneficio es mayor que el costo de estos. (Munera, 2004). Estos gastos son reconocidos dentro de unas cuentas que afecten el resultado del ejercicio, lo primero que se debe realizar bajo este método es la identificación claramente el atributo ambiental a valorar, posterior a ello se realizan observaciones directas de las medidas de mitigación reales que se realizan para proteger los efectos ambientales negativos, por último se estiman los costos de la medición para así estimar el gasto total.

Método de reposición. son los gastos para restaurar 0 devolver a su estado original el bien ambiental que ha sido afectado 0 alterado. Este método requiere la evidencia de los daños ocasionados. Para la aplicación de este método lo primero que se debe hacer es la identificación del daño ambiental que se desea valorar, mediante la realización de un análisis de personas expertas, donde se valoran a precio de mercado los insumos físicos y servicios, obteniendo el costo total a reponer por el daño y el costo por volverlo al estado inicial.

Método de la estimación del cambio de productividad. busca mediar el impacto ambiental sobre un recurso natural a través del efecto del impacto que este genera en la producción, en el costo o en las utilidades generadas, para aplicar este método se determina primero el efecto físico, mediante resultados de investigación o pruebas de laboratorio, como experimentos controlados en los cuales se inducen directamente los efectos de factores ambientales o técnicas de regresiones estadísticas. Luego se estima monetariamente dicho efecto, utilizando los precios del mercado. (Munera, 2004).
Una vez se realiza el proceso de valoración de los costos ambientales por el uso de los recursos naturales, se debe realizar el reconocimiento en la contabilidad, lo que hoy se lleva en los costos tradicionales, se hace necesario reflejar en las cuentas de contabilidad ambiental para poder evidenciar en los informes, realizar controles de los efectos de la degradación y deterioro del ambiente por el uso de recursos naturales, por ende es necesario la realización de un catálogo de cuentas que evidencien de forma detallada todas las operaciones en cuanto al impacto ambiental: para tener un control de esto se puede manejar en cuentas de orden, las cuales permiten la emisión de informes y la cuantificación de los costos ambientales.

La forma de registrar los hechos económicos referentes al reconocimiento de los costos ambientales del impacto del uso de los recursos naturales en un balance general y un estado de resultados, ha sido a través de las Cuentas de Orden que expresan los registros de la valoración del impacto ambiental por el uso de los recursos naturales. Por su naturaleza neutra las Cuentas de Orden no interfieren en la variación del patrimonio neto y sirven solamente para registrar los actos administrativos antes de sus efectos y por supuesto que el Eco-balance en este grupo de cuentas no modifica el patrimonio económico del ente, con todo, al registrar los efectos externos. (Dauzacker, 2014)

Así entonces, el patrimonio socio ambiental es la diferencia entre las ganancias y las perdidas ambientales, reflejando el efecto monetario del costo-beneficio de la utilización del medio ambiente, objetivo principal de la contabilidad ambiental. Para medir el agua es necesario tener en cuenta que esta es un elemento integrante de la riqueza natural. En la actualidad podemos visualizar la disminución del recurso agua, aunque aún tiene una dimensión geográfica, pero puede propagarse la disminución si no se pone freno al consumo, lo cual sólo puede hacerse otorgándole el carácter de bien económico.

\section{Otros Métodos}

El propósito de valorar el agua como bien económico es incentivar, vía mecanismos de precios de mercado, su uso eficiente y adecuado. Este tipo de valoraciones constituyen metodologías directas para la valoración económica de bienes y servicios ambientales (MINISTERO DE AMBIENTE VIVIENDA Y DESARROLLO TERRITORIAL, 2003) como lo es la metodología de valoración contingente (MVC). Así mismo aparecen en la guía metodologías indirectas para la valoración de bienes y servicios ambientales y recursos naturales entre las que se encuentra; 
- Enfoque hedónico

- El método de costo de viaje.

- Enfoque de producción de hogares

- Función de Daño

Finalmente, los métodos basados en costos y la técnica de transferencia de beneficios de la guía propuesta por el Ministerio, se puede afirmar que constituyen alternativas muy costosas, demandan gran cantidad de tiempo, dentro de estas metodologías, se recomienda la técnica de transferencia de beneficios como la mejor alternativa

\section{REFLEXIÓN Y POSTURA FINAL}

Todas estas disquisiciones epistemológicas se aplican perfectamente en la contabilidad ambiental, lo cual da pie para afirmar que se está en un proceso de revolución científica contable ambiental, donde es necesario establecer sistemas de medición valoración e información que den cuenta de todas estas relaciones que desde el paradigma dominante parecen inexistentes pero que el paradigma emergente desde una vertiente epistemológica normativa, se obligue a medir y valorar la naturaleza no desde la ficción que muestra la óptica rentística de los bienes y servicios ambientales sino desde el verdadero valor que deben tener estos para la vida, del tiempo que tardan en desarrollarse, del costo de reponer un bien 0 servicio que se aprovecha hoy y de las múltiples ventajas de conservar en lugar de explotar.

El costo histórico en la contabilidad ambiental, en su etapa preliminar de las relaciones entre generadores de nutrientes y de energía y consumidores primario, secundario, terciario y cuaternario (especies mayores entre ellas el ser humano), cuando ingresa al mercado, esas relaciones anteriores no son tenidas en cuenta evidenciando a todas luces un pasivo ambiental que nunca se recupera ya que el pescador por ejemplo, toma el animal y lo vende por lo que él considera que vale y que requiere para suplir otras necesidades, pero a la naturaleza jamás se le devuelven los días y meses que fueron necesarios para que el pez llegara al consumidor 0 comercializador inicial, generándose un desequilibrio en el proceso y contabilización de entradas y salidas, ya que solo sería de entradas; de tal manera que el costo histórico solo será posible de establecer de manera más o menos real cuando se trata de bienes cultivados (por la compra de alevinos por ejemplo) de resto el pasivo ambiental seguirá en aumento y hasta la compra de alevinos habría que evaluar que tan acorde es con esta realidad.

\section{CONCLUSIONES:}

Las teorías o escuelas de pensamiento en contabilidad, se consolidan desde el trabajo de Wells en 1976 alrededor de 4 grandes escuelas y una quinta como referente Hansen (1966), la Contabilidad a Valor Presente, propuestas por Solomon (July 1961) y Lemke (january, 1966). Estos métodos de valoración han sido construidos en el proceso de disputas alrededor de las anomalías que surgen en la crisis del costo histórico

Es importante distinguir que la valoración ha constituido la teoría hegemónica en contabilidad, y luego de la discusión alrededor de la crisis del costo, emerge la perspectiva multiparadigmática alternativa a la escuela hegemónica

Desde el paradigma dominante la valoración se establece solo desde el saldo, desde la cantidad de bien que ingresa al mercado, en el paradigma emergente, la valoración está integrada al reconocimiento, medición y valoración como tal del bien o servicio ambiental y del ecosistema en su conjunto. No es posible seguir valorando el árbol y su uso (la madera) sin evaluar el entorno y las múltiples relaciones del ecosistema que hacen posible que exista el árbol.

En materia de valoración contable, el costo histórico solo será posible de establecer de manera más 0 menos real cuando se trata de bienes cultivados, u otros recursos, en su ingreso al mercado. En la medida en que pretendemos valorar el agua como recurso económico, las metodologías de valoración económica, como la valoración contingente y las metodologías indirectas, son viables.

\section{REFERENCIAS:}

Chua, W. (1986). Radical Developments in Accounting Thought. The Accounting Review. Published by: American Accounting Association, 601-632, (61) 4 October.

Dane (2017). Cuenta Satélite Ambiental. En https://www.dane.gov.co/index.php/estadisticaspor-tema/ambientales/cuenta-satelite-ambientalcsa 
Demski, J. S., Fellingham, J. C., ljiri, Y., \& Sunder, S. (2002). Some Thoughts on the Intellectual Foundations of Accounting. Accounting Horizons, 157-168.

Franco, R. (2018) Contabilidad de recursos naturales. En. https://www.youtube.com/watch?v=-pUOJj74_9Y

Glover, J. I. (2008). Separación de los hechos y los pronósticos en los estados financieros. Innovar, 18(31), 135-146.

Goméz, A. (2018). Contabilidad ambiental en un ecosistema de manglar. Caso delta del Río Atrato, Urabá Antioqueño. Tesis de maestría en Medio Ambiente y Desarrollo Sostenible. Unal de Colombia sede Medellín. Candidata.

Gomez, M. (2011). Pensando los fundamentaos de la disciplina contable. Lumina, 120 - 150.

Lee, A. (2009). The Ontology and Epistemology of Social Reality in Accounting According to Mattessich. Accounting and Public Interest, 9(1), 65-72.

Macinstosh, N., Sheare, T., Thorton, D., \& Welker, M. (2000). Accounting as simulacrum and hyperreality: perspectives on income and capital. Accounting, Organization and Society, 13-50.

Machado, M. (16 de 09 de 2018). Modelo contable en un manglar (G. Alba, Entrevistador)

Mattessich, R. (2003). Accounting representation and the onion model of reality: a comparison with Baudrillard's orders of simulacra and his hyperreality. Accounting, Organization and society(28), 443-470.

Mercado-Domenech, S. (2008). Capitulo 1. Aspectos Fundamentales de la ciencia. Parte 1 Estadistica y ciencia. En H. Perez-Tejada, Estadística para las ciencias sociales, del comportamiento y la salud (págs. 3-16). México D.F.: Cengage Learning.
MINISTERO DE AMBIENTE VIVIENDA Y DESARROLLO TERRITORIAL. (2003). METODOLOGIA DE VALORACION. Obtenido de http://www.minambiente.gov.co/images/Negocios Verdesysostenible/pdf/569_guiavaloracion.pdf

Muñoz-Restrepo, C. (2018). Los Juicios Precientificos en el Metodo Contable. Medellin.

Naciones Unidas. (2016). Sistema de Contabilidad Ambiental y Económica (SCAE) 2012. New York, Estados Unidos: NU.

Neimark, M. (1990). The King is Dead.LongLive the King! Critical Perspectives on Accounting(1), 103-114.

Osorio, J. (2014). La Nocion de Inconmesurabilidad en Thomas Kuhn. Recuperado el 2017, de http://www.ugr.es/ perisv/docen/asigna/curmono hfc/alum\%20trabajos/2013-

2014/La\%20nocion\%20de\%20inconmensurabilid ad\%20en\%20Thomas\%20S.\%20Kuhn\%20JAVIE R\%200SORIO.pdf

Riahi-Belkaoui, A. (2000). Cap 10 the events and behavioral approaches. En A. Riahi-Belkaoui, Accounting Theory (págs. 297-330). London: Thompson Learning.

Ritzer, G. (Aug., 1975). Sociology: A Multiple Paradigm Science. The American Sociologist, 10(3), 156167.

Tarski, A. (Diciembre de 1999). La Concepcion Semantica de Verdad (Trad. Paloma Garcia Abad). A Parte Rei Revista de Filosofia(6). Recuperado el 28 de Octubre de 2018, de www.ucm.es/info/pslogica/verdadtarski.pdf: http://serbal.pntic.mec.es/ cmunoz11/page14.ht $\mathrm{ml}$

Wells, M. C. (Jul., 1976). A Revolution in Accounting Thought? The Accounting Review, 51(3), pp. 471-482. 